\title{
Três décadas de StaGe-Gate: AnÁlise da Evolução do Modelo
}

Achiles Camilo Soares Neto (achiles@usp.br) - Universidade de São Paulo (USP).

Vitória Pereira Pinto (vitoriapereirap@usp.br) - Universidade de São Paulo (USP).

Roberto Marx (ㅁobemarx@usp.br) - Universidade de São Paulo (USP).

Mario Sergio Salerno (․msalerno@usp.br) - Universidade de São Paulo (USP).

\section{RESUMO}

O modelo Stage-Gate foi inicialmente apresentado em 1990 e, após três décadas, ainda possui significativa influência nos estudos de gestão da inovação e desenvolvimento de novos produtos. Este estudo apresenta uma síntese de como o modelo Stage-Gate evoluiu ao longo dos anos, através de análise das publicações de seu criador, Robert G. Cooper. São apresentados seis momentos importantes que são fundamentais para entender como o modelo foi criado e como se mantém relevante. Há diversas críticas sobre a rigidez do Stage-Gate, mas por vezes essas se referem à primeira versão do modelo. O Stage-Gate está constantemente sendo aperfeiçoado. Após uma sequência de adaptações e flexibilizações, a versão mais atual do modelo, publicada em 2018, incorpora práticas ágeis e é renomeado para Agile-Stage-Gate. Cooper sustenta um fluxo de publicações evoluindo seu processo, o mantendo atualizado e em aderência às necessidades de mercado.

Palavras-chave: stage-gate; desenvolvimento de novos produtos; modelos de inovação 


\section{INTRODUÇÃO}

O modelo de Stage-Gate é um mapa conceitual e operacional para mover projetos de novos produtos da ideia para o lançamento e além - um blueprint para gerenciar o processo de novos produtos (NPD) para melhorar eficiência e eficácia. O processo de inovação pode ser visualizado como uma série de estágios, com cada estágio composto por um conjunto de atividades recomendadas ou mandatórias de boas práticas necessárias para o progresso do projeto para o próximo 'gate' ou ponto de decisão. Seguindo cada estágio está um 'gate' ou um ponto de decisão Go/Kill (COOPER, 2008).

Esse modelo simplificado foi apresentado por Cooper (1990) e é uma das principais abordagens utilizadas para gerenciamento de inovação e desenvolvimento de novos produtos (SALERNO et al., 2015). Este modelo foi atualizado pelo próprio autor em sucessivas ocasiões (COOPER, 1994, 2008, 2014; COOPER; SOMMER, 2018; COOPER; EDGETT; KLEINSCHMIDT, 2002a, 2002b). Entretanto, há diversas críticas ao modelo que, por vezes, se baseiam em sua primeira versão, o que motivou o próprio autor a "se defender" através de algumas publicações (COOPER, 1994, 1996, 2014; EDWARDS et al., 2019).

Visando dar clareza à acadêmicos e praticantes, esse estudo almeja apresentar uma análise consolidada e concisa de como o modelo Stage-Gate evoluiu, quais são suas diferentes versões e como se mantém relevante após 30 anos.

\section{METODOLOGIA}

Para analisar como o modelo Stage-Gate evoluiu desde sua criação, segundo o próprio autor, foi realizada a busca na base de dados Scopus por "stage-gate" e filtrado artigos com o autor “Cooper, R.G.", o que resultou em um total 26 documentos. Os mesmos termos e critérios foram utilizados na base de dados Web of Science, porém, essa resultou em apenas 22 documentos, fazendo assim com que os autores dessem preferência à Scopus. Adicionalmente, foram adicionados três trabalhos de alta relevância do próprio autor que não constaram como resultado de busca, mas se apresentaram como essenciais para entendimento da evolução do modelo. Estes estudos são dois artigos prévios à formalização do modelo estudado (COOPER, 1985; COOPER; KLEINSCHMIDT, 1988).

Foi realizada a análise de conteúdo dos 29 documentos presentes da base e os pontos mais relevantes para análise de como o modelo Stage-Gate evoluiu são apresentados na seção de resultados. 


\section{RESULTADOS}

\subsection{Estudos prévios ao Stage-Gate}

Inicialmente, Cooper (1985) propôs um modelo chamado NewProd System em contraponto às ineficiências dos modelos vigentes para screening de projetos de novos produtos. Visando cobrir a carência presente na época, o NewProd System foi apresentado como um modelo de scoring para pontuação de projetos que foi construído com uma base histórica de 200 projetos e obteve uma assertividade de $84 \%$. Com esse novo modelo, Cooper ambicionou ofertar para os praticantes um mecanismo que fomentasse maior eficiência no processo de inovação, através de uma ferramenta de apoio ao screening. Entretanto, este se limita somente à "fase" de seleção, todas as demais fases do processo não são contempladas no estudo, apesar de terem sido criticadas pelo autor.

Posteriormente, ao terminar o estudo com os 200 projetos que originaram o artigo anterior, novos resultados são apresentados por Cooper e Kleinschmidt (1988). Neste estudo, são analisadas as alocações de recursos entre os diversos estágios do projeto, onde Cooper se aprofunda e detalha de como os custos se dividem em diversas atividades dentro do processo de Desenvolvimento de Novos Produtos (DNP). Para análise comparativa, os passos são agrupados em três grandes estágios (descritos na legenda da Figura 1).

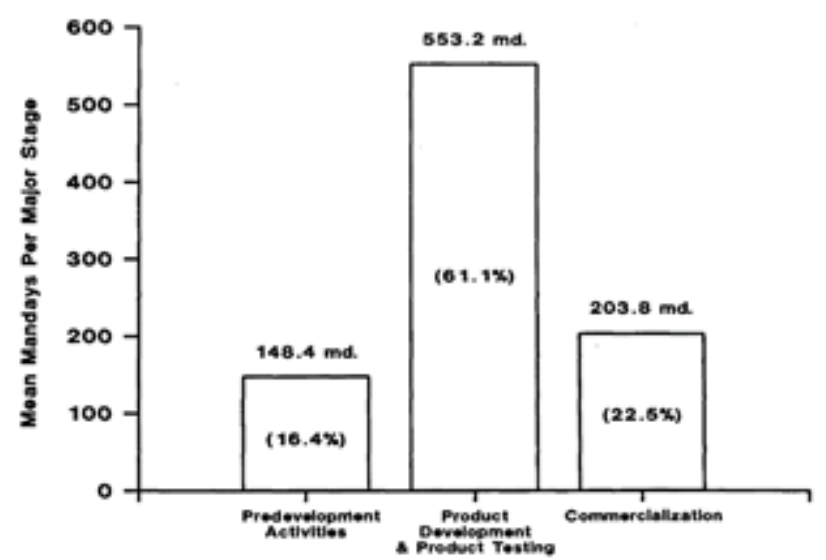

FIGURA 1 - Percentual de gastos divididos por grandes estágios no processo de DNP. Fonte: Cooper e Kleinschmidt (1988)

Através da clara divisão em macro estágios, e concluindo que análises intermediárias entre os estágios seriam fundamentais para evitar um gasto desnecessário, ao final do estudo é recomendado que sejam criados 'gates'. Somado à contribuição apresentada no artigo de 1985, Cooper apresenta uma proposição para uma seleção mais assertiva de projetos de inovação e sugere que o praticante defina o processo. No estudo de 1988, há uma análise de onde estão os maiores gastos por macro estágio e uma proposição para que o praticante estabeleça pontos de 
decisão entre tais. Neste momento, Cooper apresenta informações suficientes para que o leitor estruture um processo de maneira adequada, sem uma delimitação ou proposição de qual seria o melhor modelo a ser seguido.

\subsection{Criação do Stage-Gate (Segunda geração)}

Embasado nas proposições feitas aos praticantes (COOPER, 1985; COOPER; KLEINSCHMIDT, 1988), é feita uma construção de um processo para gestão de novos produtos, se propondo a cobrir uma deficiência do mercado, que possuía uma alta taxa de falhas e baixos resultados. Cooper (1990, 1993) apresenta o Stage-Gate System como uma solução para melhorar o gerenciamento das atividades de desenvolvimento de novos produtos, incluindo o elemento de decisão ('gates') entre as fases do processo ('stages'). Segundo Cooper (1994), a primeira geração do Stage-Gate foi o NASA PPP (Phased Project Planning), hoje conhecido como Phased Review Process, que foi desenvolvido na década de 1960. Este modelo criado pela NASA (National Aeronautics and Space Administration) apresenta a divisão por fases e possui pontos de revisão ao final de cada uma delas, verificando o prazo e a completude das atividades. Entretanto, esse modelo prevê uma abordagem voltada ao monitoramento e controle, além de ser direcionada aos estágios de design físico e desenvolvimento do produto, não contemplando marketing/vendas ou riscos de negócio. O modelo Stage-Gate de Cooper foi inicialmente apresentado no artigo "Stage-gate systems: A new tool for managing new products" (COOPER, 1990) e posteriormente na segunda edição do livro "Winning at New Products" (COOPER, 1993).

Com o sistema de Stage-Gate (Figura 2), Cooper propõe uma visão holística e integrada do processo, em que o marketing/vendas é parte integrante, direcionando uma abordagem voltada ao mercado, além de apresentar uma maior ênfase ao trabalho de pré-desenvolvimento do produto, que é dado como feito no modelo anterior (NASA PPP). Adicionalmente, Cooper apresenta como diferencial a possibilidade de paralelização de atividades, mas não o paralelismo de estágios, mantendo limitações quanto à flexibilidade do processo.

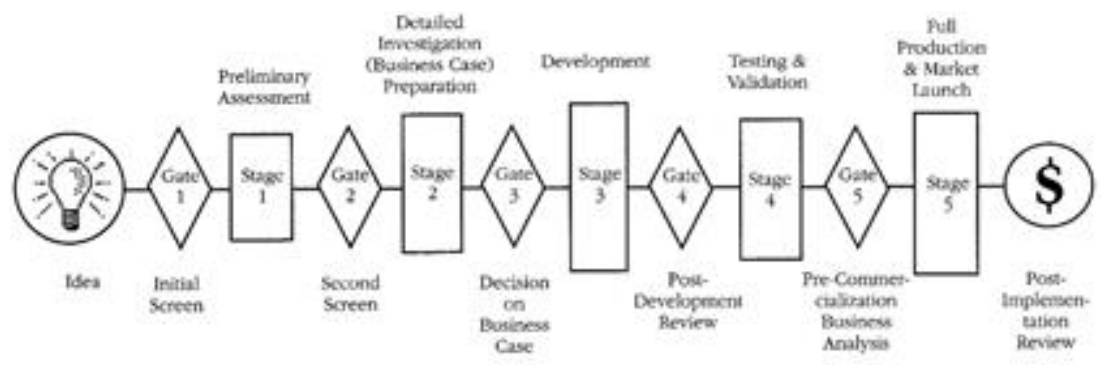

FIGURA 2 - Primeira versão do Stage-Gate. Fonte: Cooper (1990) 
O sistema Stage-Gate de segunda geração possui 5 'stages' e 5 'gates': Gate 1 - Screen inicial; Stage 1 - Avaliação preliminar; Gate 2 - Segundo screen; Stage 2 - Definição (do Business Case); Gate 3 - Decisão sobre o Business Case; Stage 3 - Desenvolvimento; Gate 4 - Revisão pós-desenvolvimento; Stage 4 - Validação (e Teste do produto); Gate 5 - Decisão précomercialização; Stage 5 - Comercialização.

\subsection{Primeira evolução do modelo}

Cooper (1994) apresenta uma nova versão do Stage-Gate Systems otimizando o processo para obtenção de eficiência. São adicionados quatro "F" fundamentais: fluidez, flexibilidade, foco e fuzzy gates (decisões condicionais). A terceira geração caracteriza-se por sua adaptabilidade a diferentes situações, uma vez que não é estritamente sequencial comparando-se com a segunda geração. Neste novo modelo, algumas atividades com duração prolongada podem ser antecipadas na terceira geração do Stage-Gate System, porém este recurso deve ser feito conscientemente a fim de evitar prejuízos ao desenvolvimento do projeto. Com essa possibilidade, há uma descentralização da tomada de decisão, fornecendo maior autonomia à liderança operacional e ao time de projeto.

O paralelismo se torna a evolução mais visível ao ser analisada a representação gráfica do modelo (Figura 3). Entretanto, a inserção de fuzzy gates, caracterizada por mais de um losango interconectado, provoca uma mudança significativa entre as duas gerações do sistema. Ao inserir a possibilidade de decisões condicionais e situacionais, é inserida agilidade no processo. A fluidez adquirida por permitir que decisões sejam feitas em situações específicas e/ou com determinadas condições, possibilita que o fluxo siga sem travas inerentes ao processo que demandam que todas as atividades fossem concluídas antes do processo mover para ao estágio subsequente. Contudo, tamanha flexibilização pode promover uma "desgovernança", caso as atividades, condições e situações sejam devidamente controladas, evitando que não haja uma descaracterização do processo.

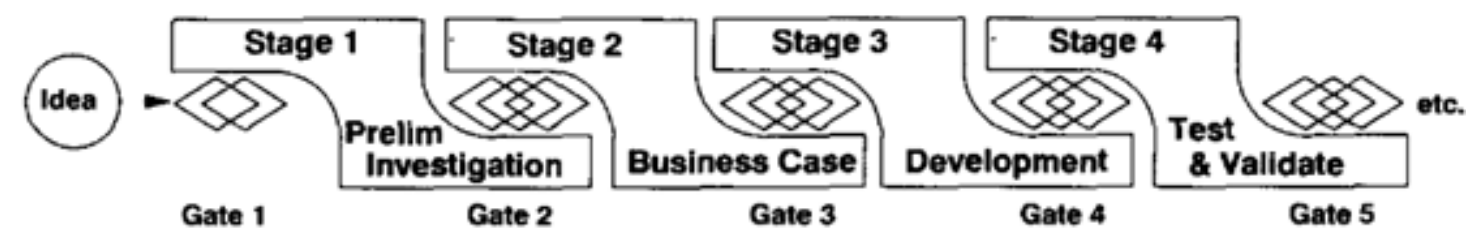

FIGURA 3 - Terceira geração do processo de Stage-Gate. Fonte: Cooper (1994)

\subsection{Uma visão de portfólio}


Segundo Cooper, Edgett e Kleinschmidt (2002a), 60\% das empresas entrevistadas pelo PDMA (Product Development \& Management Association) usam alguma adaptação mais sofisticada da terceira geração do Stage-Gate para o desenvolvimento de novos produtos. Portanto, Cooper, Edgett e Kleinschmidt (2002a, 2002b) publicam dois estudos que buscam responder o que mais as empresas têm feito para aumentar a eficácia de seu novo processo de produto, por meio de incrementos e melhorias na terceira geração do Stage-Gate.

Dentre as melhores práticas encontradas por Cooper, Edgett e Kleinschmidt (2002a) destaca-se a adição de um estágio de descoberta ao início do processo para gerar ideias inovadoras de produtos de forma muito mais proativa. Este modelo apresenta um processo de cinco estágios que incorpora um estágio de 'Descoberta' (Figura 4). Anteriormente, antes de dar início ao processo tradicional do Stage-Gate, era necessário que produto e o projeto já tivessem sido definidos (COOPER, 2014).

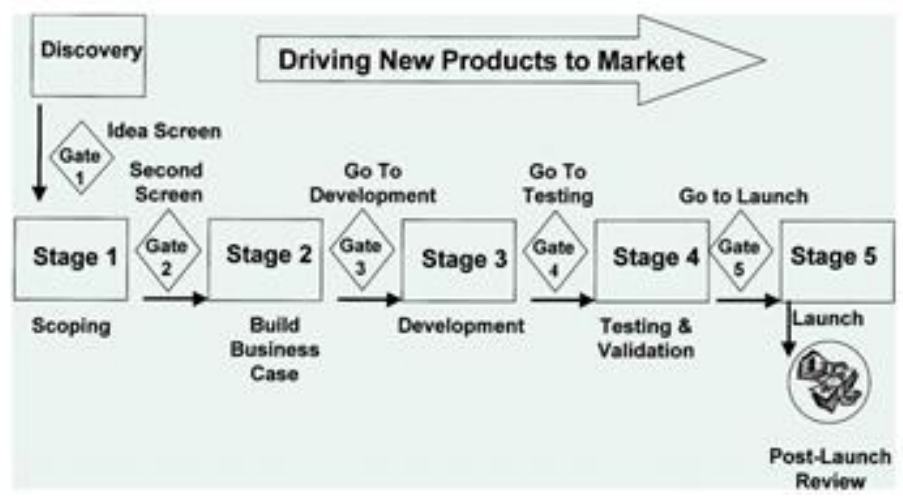

FIGURA 4 - Incorporação do estágio de "Descoberta" no início do processo de Stage-Gate. Fonte Cooper,

Edgett e Kleinschmidt (2002a)

O incremento desse novo estágio chamado de 'Descoberta' visa aprimorar o fluxo de ideias e a captação de novas oportunidades no início do processo. Novas perspectivas e sugestões para melhor se trabalhar com esse novo cenário são apresentadas, como escutar a voz do cliente (VoC), realizar campanhas com os clientes atuais, trabalhar com maior proximidade aos clientes líderes, além de simular diferentes cenários. Tais práticas, visam fomentar que a ideia seja trabalhada e amadurecida antes de ser iniciado o primeiro estágio.

De maneira complementar, Cooper, Edgett e Kleinschmidt (2002b) flexibiliza o processo para diferentes tamanhos de projetos. Almejando adaptar o sistema para que comporte a atuação com portfólio, é apresentado novos modelos mais enxutos e simplificados que possibilitam assim, o acompanhamento e controle dos projetos em um processo único, que se acomoda a três diferentes configurações, contemplando assim demandas e ideias menores, sem que haja o rigor 
de percorrer todos os 5 estágios e 'gates' previstos no modelo original (Figura 5). Adicionalmente, também é apresentado casos práticos onde a condução dos 'gates' foi flexibilizada.

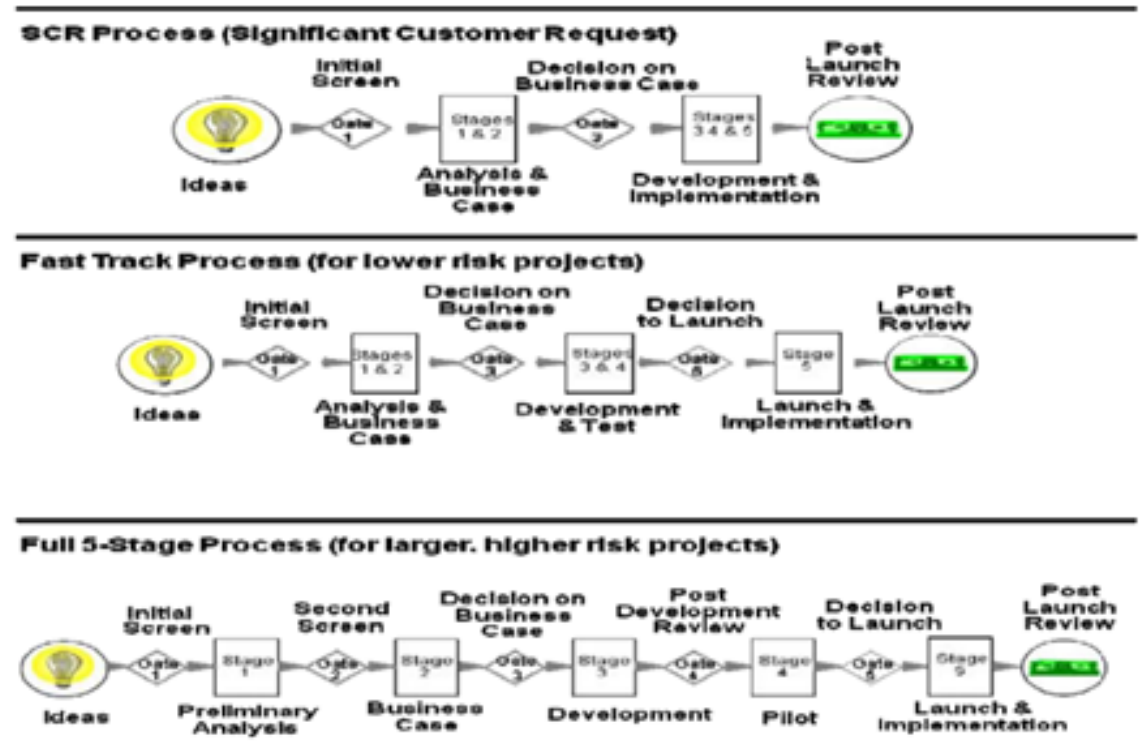

FIGURA 5 - Novos processos simplificados como flexibilização do modelo tradicional do Stage-Gate. Fonte: Cooper, Edgett e Kleinschmidt (2002b)

Estes incrementos apresentados tornam o processo mais robusto, permitindo o acompanhamento de todas as iniciativas de inovação em um único macrofluxo, e mais inteligente, ao incluir as devidas flexibilizações para diferentes contextos e promover um amadurecimento da ideia prévio aos estágios iniciais, mas ainda mantendo essa fase no processo, para efeitos de controle. Desta forma, a visão holística do fluxo é ampliada.

\subsection{Stage-gate flexível (Ágil)}

Cooper (2008) apresenta de maneira estruturada as novas versões do Stage-Gate introduzidas nos artigos de 2002, às tornando ainda mais flexíveis e adaptáveis para lidar com muitos tipos e tamanhos diferentes de projetos. O formato 'Fast Track Process' se torna Stage-Gate XPress, para projetos de risco moderado, como melhorias, modificações e extensões; e o 'SCR Process' se torna Stage-Gate Lite para projetos muito pequenos, como solicitações simples de clientes (Figura 6). Percebe-se, portanto, que apesar de todos os projetos apresentarem riscos, consumirem recursos e possuírem a necessidade ser gerenciados, nem todos precisam seguir o fluxo do Stage-Gate tradicional. Adicionalmente, é evidenciada a fase de Descoberta como ponto de partida para todos os processos. 


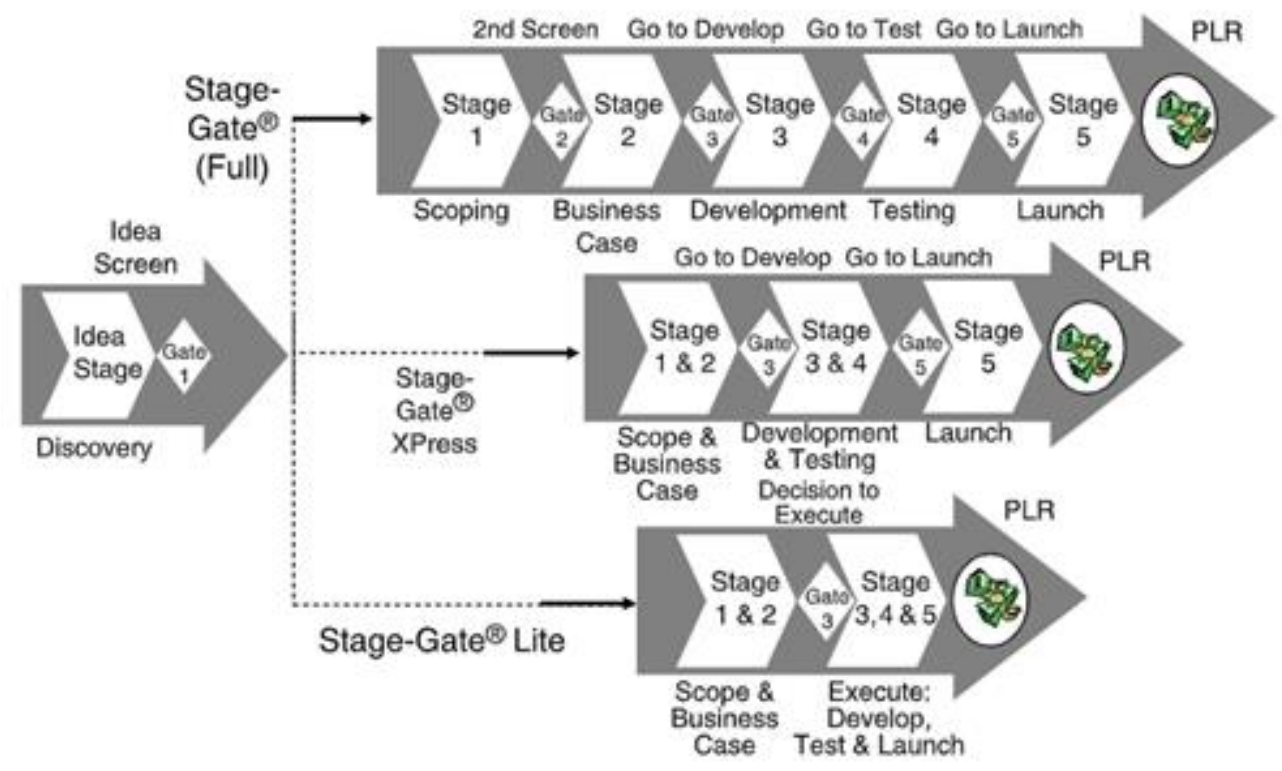

FIGURA 6 - Processo 'Next Generation Stage-Gate’. Fonte: Cooper (2008)

Almejando tornar o processo ainda mais adaptável, Cooper (2008) cria um modelo em espiral e incorpora melhores práticas de tomada de decisão, como scorecards e gestão de melhoria contínua, durante a revisão pós-lançamento. O autor agrega ao seu modelo características de desenvolvimento ágil, ambicionando obter maior rapidez no processo, para finalizar o produto por meio de uma série de interações "build-test-feedback-and-revise" (COOPER, 2008).

O sistema de espirais se acopla bem ao conceito ágil de sprints, onde alguma versão do produto pode ser apresentada ao final de cada ciclo às partes interessadas - clientes e empresa (COOPER; SOMMER, 2016). Dentre os benefícios deste modelo híbrido, destaca-se a estrutura para lidar com incertezas no front-end, a maior velocidade do processo através da adoção de iterações, e a possibilidade de obter uma maior taxa de sucesso dos novos produtos, uma vez que o modelo requer que a equipe do projeto interaja constantemente com os usuários e clientes (Figura 7). 


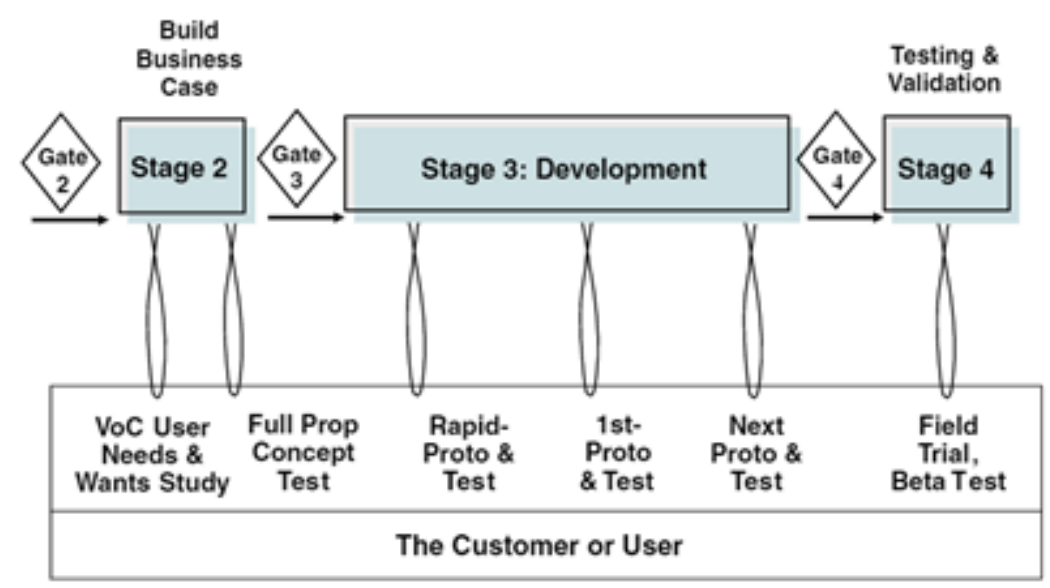

FIGURA 7 - Desenvolvimento em espiral como uma série das iterações de "Build-Test-Feedback-Revise".

Fonte: Cooper (2008)

Complementarmente, Cooper agrega ao seu modelo características de um sistema de inovação aberta (open innovation), se adequando à realidade observada pelo autor em diversas companhias de grande porte (Figura 8). Desta forma, o sistema Stage-Gate visa construir a flexibilidade necessárias a fim de permitir maior aderência à necessidade de conectar à rede de parceiros, alianças e fornecedores, desde a geração de ideias até o lançamento. Ao fazer isso, maior valor é criado e obtido ao longo do processo.

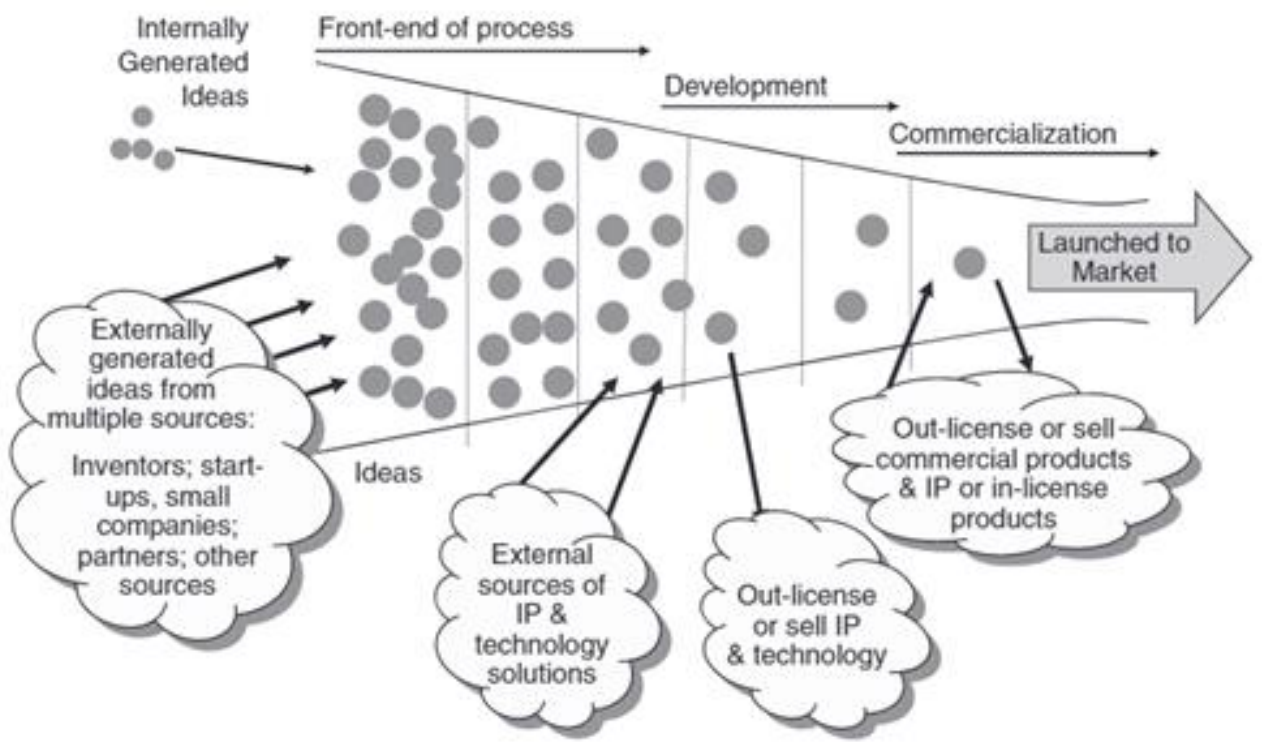

FIGURA 8 - Stage-Gate adaptado para inovação aberta. Fonte: Cooper (2008) 


\subsection{O que vem depois do Stage-Gate?}

Cooper (2014) revisa o modelo, complementando o método de espiral (apresentado em 2008) com o "Sistema Triplo A", um sistema flexível, ágil e acelerado (Figura 9). Desta forma, almeja-se uma melhora em relação à adaptabilidade e flexibilidade do sistema, que incorpora a estratégia do desenvolvimento em espiral para apresentar resultados tangíveis aos clientes com maior frequência, através de "sprints", que possuem curtas durações e não exigem padrões de “go/kill” rígidos.

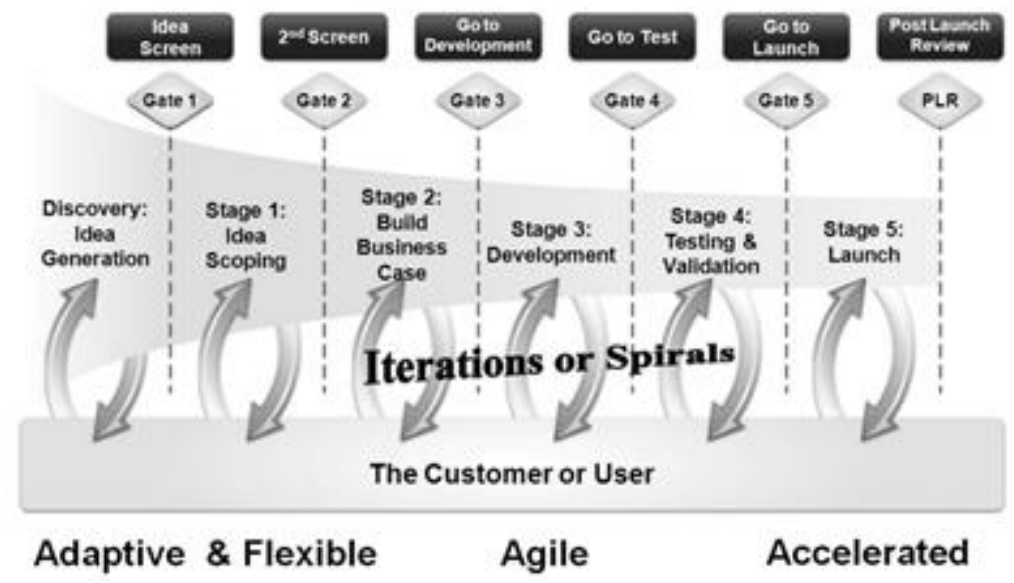

FIGURA 9 - Nova geração do sistema 'idea-to-launch'. Fonte: Cooper (2014)

Antes dessa adaptação, o método requeria que o produto e o projeto estivessem definidos antes de o projeto passar para o desenvolvimento (COOPER, 2014). O modelo de espiral permite com que os times continuem agregando feedbacks de valor do cliente para dentro do produto a qualquer momento do desenvolvimento. De acordo com Cooper (2014), as atividades esperadas em cada ciclo de uma espiral consistem em "construir", "testar", "obter feedback", "revisar". Cada um desses loops contribui para que o produto se aproxime cada vez mais da sua versão final, e que obtenha maior chance de ser bem aceito pelo mercado quando for lançado, devido aos feedbacks constantes (Figura 10). 


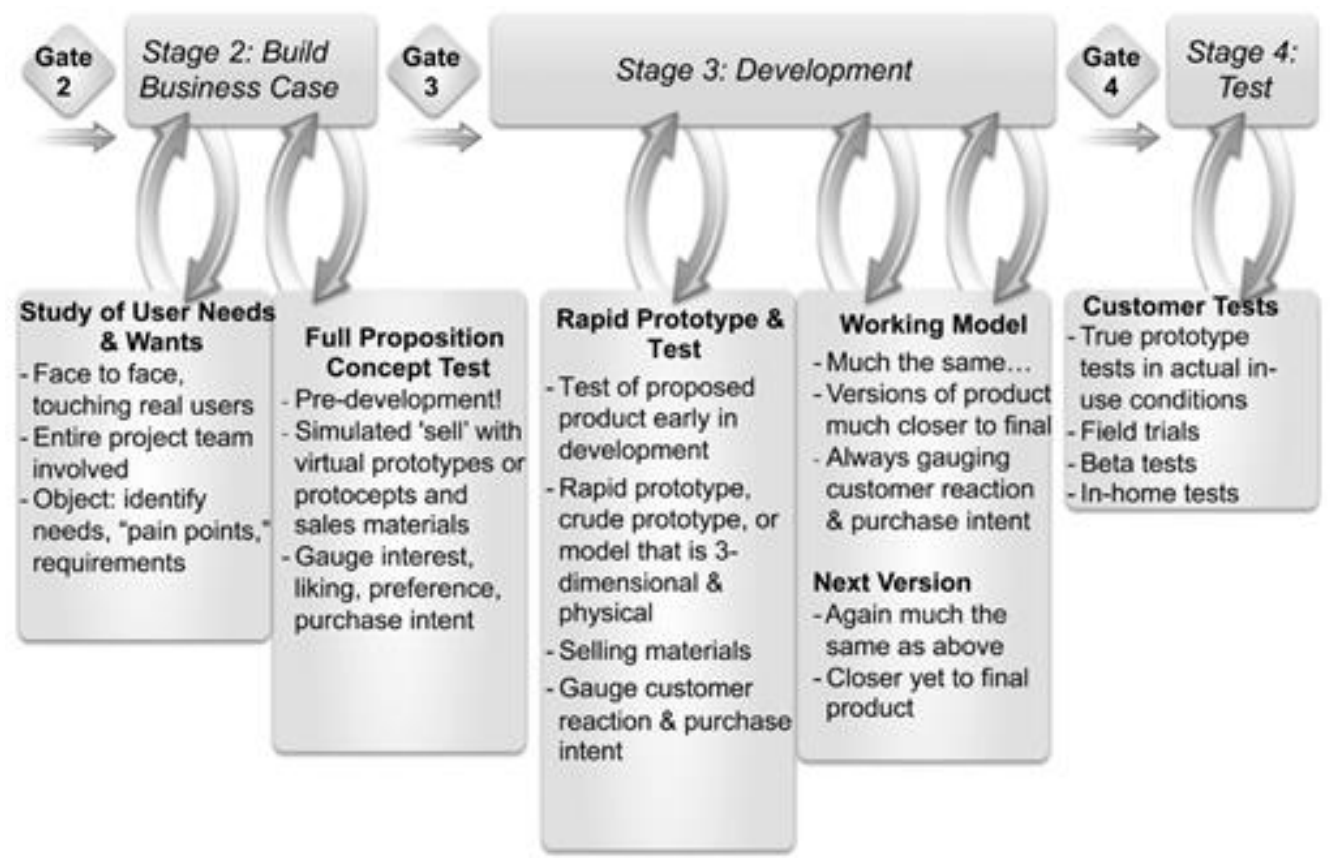

FIGURA 10 - Desenvolvimento em espiral em cima de iterações de 'build-test-feedback-revise'. Fonte: Cooper

Desde o primeiro flerte com abordagens ágeis em 2008, Cooper se aproximou cada vez mais de um modelo híbrido em suas publicações subsequentes (COOPER, 2008, 2014, 2016; COOPER; SOMMER, 2016, 2018, 2020; EDWARDS et al., 2019). Em sua última atualização, Cooper e Sommer (2018) apresenta o modelo como uma abordagem híbrida chamada AgileStage-Gate, prevendo uma completa integração entre os estágios com as práticas presentes nas metodologias ágeis (Figura 11).

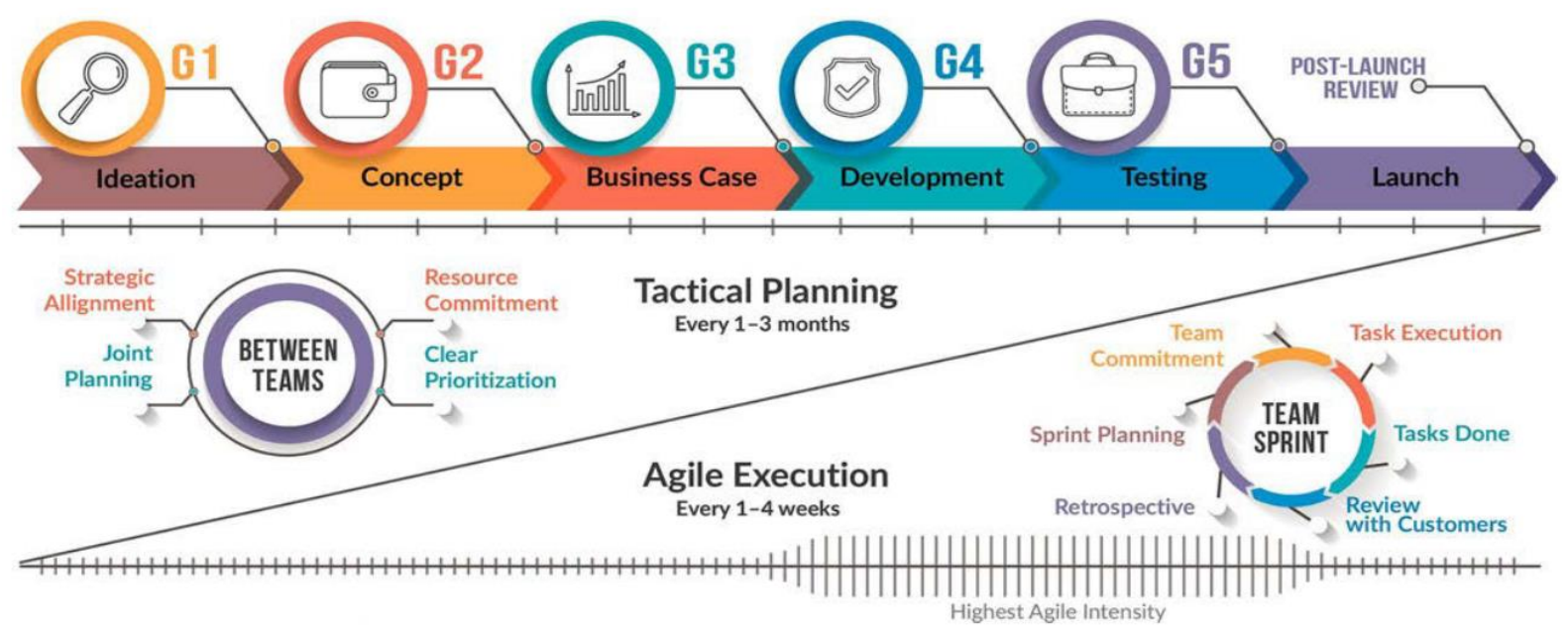

FIGURA 11 - Modelo híbrido Agile-Stage-Gate. Fonte: Cooper e Sommer (2018) 


\section{DISCUSSÃO}

Cooper (2014) diz que o Stage-Gate atraiu uma série de críticas, sendo tido como um método muito linear e rígido. A exemplo destas críticas, destaca-se o texto publicado por Salerno et al. (2015), que desafia os modelos tradicionais de gestão da inovação, dentre eles o modelo de Stage-Gate, principalmente pelo fato deles seguirem uma sequência linear e pré-definida de fases: geração de ideias, seleção de ideias, desenvolvimento e lançamento ao mercado. Os autores afirmam que, embora os modelos tradicionais continuem a ser influentes, eles podem não se encaixar bem em determinados ambientes, como em situações de inovação radical e entrada em novos mercados.

Em contrapartida, Cooper (2008) defende que o Stage-Gate não é uma ferramenta estática, mas, sim, um sistema abrangente e mutável, que se baseia em muitas das melhores práticas e métodos e que está sempre se transformando. $\mathrm{O}$ autor afirma que, apesar de ser um método para transformar uma ideia em um novo produto de sucesso, muitos desvios podem ser feitos nesse "meio do caminho". Uma das vantagens do método é ser adaptável às circunstâncias das empresas, ou seja, algumas etapas podem ser puladas, sobrepostas, as atividades podem ocorrer em paralelo e dentro de cada estágio não há uma linearidade de tarefas, cabendo ao time do projeto criar seu próprio plano. Portanto, o processo não é linear, mesmo que os gráficos apresentem isso.

Adentrando nas evoluções do modelo do Stage-Gate, ao contrário do método inicial desenvolvido pela NASA (Phased Review Process), no qual cada estágio do processo de inovação se reporta a um determinado departamento, no Stage-Gate os estágios são multifuncionais, a fim de trazer rapidez na entrega. Nesse sentido, Cooper (2008) afirma que o Stage-Gate é um processo de negócios, não um processo limitado a um departamento como P\&D ou Marketing. O objetivo de Cooper (1990) ao criar o Stage-Gate, como uma segunda geração evoluída a partir do modelo da NASA, era de melhorar o gerenciamento das atividades de desenvolvimento de produtos.

Posteriormente, Cooper (1994) insere flexibilidade ao processo, prevendo o paralelismo entre as fases, o que estava restrito no modelo anterior, que permitia somente concorrência de atividades. Para viabilizar que duas fases fossem executadas em paralelo, o conceito de fuzzy gates é introduzido, permitindo que as tomadas de decisão para evolução entre estágios sejam condicionais ou situacionais. Essa evolução do sistema Stage-Gate é fundamental para que haja maior aderência à realidade do mercado e traz uma relevância significativa para que o modelo atenda às necessidades reais. 
Desde a criação da sua terceira geração, o Stage-Gate passou por diversas evoluções sempre no sentido de torná-lo mais adaptável e flexível. Em Cooper, Edgett e Kleinschmidt (2002a) foi apresentado um estudo das práticas e adaptações feitas pelas empresas nos sistemas de desenvolvimento de produtos. Percebe-se que as práticas avaliadas se parecem com o processo tradicional, ou seja, apesar dos incrementos e melhorias propostas, os aspectos de estágios e 'gates' permanecem imutáveis. Entretanto, surgem processos de geração mais ágeis, dinâmicos e flexíveis, mais enxutos, mais rápidos e mais adaptáveis (COOPER, 2008, 2014).

\section{CONCLUSÃO}

Inicialmente, abordando o método do Stage-Gate de maneira geral, uma das principais características deste modelo, desde sua criação, é o conceito de 'gates', os quais servem de ponto de decisão ou bloqueio de um determinado projeto. Com isso, uma boa definição dos 'gates' pode ser determinante para um sucesso de um projeto e para a vantagem competitiva da empresa, que poderá se antecipar e descartar rapidamente projetos com maior probabilidade de insucesso. Nesse sentido, o Stage-Gate se tornou um processo escalonável para se adequar a diferentes tamanhos de projetos e níveis de riscos diferentes, acoplando com diversas necessidades de mercado (COOPER, 2008; COOPER; EDGETT; KLEINSCHMIDT, 2002b). De maneira complementar, Cooper (2008) agregou ao modelo o conceito de desenvolvimento em espiral ou ágil, que busca permitir às equipes de projeto avançarem de forma mais rápida para a finalização do projeto do produto por meio de iterações de "build-test-feedback-andrevise" com contato constante com o cliente. Adicionalmente, o modelo foi modificado para acomodar a inovação aberta, buscando se atualizar para atender novas tendências.

Cooper (2008) reforça que o processo de Stage-Gate está sendo constantemente aperfeiçoado pelas próprias empresas que usam o modelo. Dessa forma, o sistema se torna mais atual, mantém sua relevância e permite uma evolução natural via acoplamentos de outros modelos e métodos de gerenciamento que promovem a continuidade do uso do modelo.

O sistema Stage-Gate está intrinsecamente ligado a seu autor, que desde sua criação até 30 anos depois, o mantém constantemente atualizado. A estrutura simples de 5 'stages' e 5 'gates' evoluiu significativamente ao longo do tempo, mas continua atendendo demandas existentes de mercado e alimentando o desenvolvimento de novas teorias relacionadas a processos de inovação. Sua relevância eminente se dá pela facilidade de identificar seu uso em cenários reais, 
que inclusive são apresentados continuamente pelo próprio autor (COOPER, 2021; COOPER; SOMMER, 2020; EDWARDS et al., 2019).

\section{AGRADECIMENTOS}

A pesquisa foi parcialmente financiada pela Fapesp - Fundação de Amparo à Pesquisa do Estado de São Paulo, processo 2015/26662-5.

\section{REFERÊNCIAS}

COOPER, Robert G. Perspective third-generation new product processes. Journal of Product Innovation Management, [S. l.], v. 11, n. 1, p. 3-14, 1994. DOI: 10.1016/0737-6782(94)90115-5.

COOPER, Robert G.; KLEINSCHMIDT, E. J. Resource allocation in the new product process. Industrial Marketing Management, [S. l.], v. 17, n. 3, p. 249-262, 1988. DOI: 10.1016/0019-8501(88)90008-9.

COOPER, Robert G. Selecting winning new product projects: using the NewProd System. Journal of Product Innovation Management, [S. l.], v. 2, n. 1, p. 34-44, 1985. DOI: 10.1111/1540-5885.210034.

COOPER, Robert G. Stage-gate systems: a new tool for managing new products. Business Horizons, [S. l.], v. 33, n. 3, p. 44-54, 1990. DOI: 10.1016/0007-6813(90)90040-I.

COOPER, Robert G. Winning at New Products. Second Edi ed. [s.l.] : Addison-Wesley Publishing Company, 1993.

COOPER, Robert G. Overhauling the new product process. Industrial Marketing Management, [S. l.], v. 25, n. 6, p. 465-482, 1996. DOI: 10.1016/S0019-8501(96)00062-4.

COOPER, Robert G. Perspective: The Stage-Gate ${ }^{\circledR}$ idea-to-launch process-update, what's new, and nexgen systems. Journal of Product Innovation Management, [S. l.], v. 25, n. 3, p. 213-232, 2008. DOI: 10.1111/j.1540-5885.2008.00296.x.

COOPER, Robert G. What's next?: after stage-gate. Research-Technology Management, [S. l.], v. 57, n. 1, p. 20-31, 2014. DOI: $10.5437 / 08956308 X 5606963$.

COOPER, Robert G. Agile-stage-gate hybrids. Research-Technology Management, [S. l.], v. 59, n. 1, p. $21-$ 29, 2016. DOI: $10.1080 / 08956308.2016 .1117317$.

COOPER, Robert G. Accelerating innovation: some lessons from the pandemic. Journal of Product Innovation Management, [S. l.], v. 38, n. 2, p. 221-232, 2021. DOI: 10.1111/jpim.12565.

COOPER, Robert G.; EDGETT, Scott J.; KLEINSCHMIDT, Elko J. Optimizing the stage-gate process: what bestpractice companies do-I. Research-Technology Management, [S. l.], v. 45, n. 5, p. 21-27, 2002. a. DOI: 10.1080/08956308.2002.11671518.

COOPER, Robert G.; EDGETT, Scott J.; KLEINSCHMIDT, Elko J. Optimizing the stage-gate process: what bestpractice companies do-II. Research-Technology Management, [S. l.], v. 45, n. 6, p. 43-49, 2002. b. DOI: 10.1080/08956308.2002.11671518.

COOPER, Robert G.; SOMMER, Anita F. The Agile-Stage-Gate Hybrid Model: A promising new approach and a new research opportunity. Journal of Product Innovation Management, [S. l.], v. 33, n. 5, p. 513-526, 2016. DOI: $10.1111 /$ jpim.12314.

COOPER, Robert G.; SOMMER, Anita Friis. Agile-stage-gate for manufacturers. Research-Technology Management, [S. l.], v. 61, n. 2, p. 17-26, 2018. DOI: 10.1080/08956308.2018.1421380.

COOPER, Robert G.; SOMMER, Anita Friis. New-product portfolio management with agile. ResearchTechnology Management, [S. l.], v. 63, n. 1, p. 29-38, 2020. DOI: 10.1080/08956308.2020.1686291.

EDWARDS, Kasper; COOPER, Robert G.; VEDSMAND, Tomas; NARDELLI, Giulia. Evaluating the agilestage-gate hybrid model: experiences from three SME manufacturing firms. International Journal of Innovation and Technology Management, [S. l.], v. 16, n. 08, p. 1950048, 2019. DOI: 10.1142/S0219877019500482. 
SALERNO, Mario Sergio; GOMES, Leonardo Augusto de Vasconcelos; SILVA, Débora Oliveira da; BAGNO, Raoni Barros; FREITAS, Simone Lara Teixeira Uchôa. Innovation processes: which process for which project? Technovation, [S. l.], v. 35, p. 59-70, 2015. DOI: 10.1016/j.technovation.2014.07.012. 